\title{
Incidence and radiological characteristics of fabellae in an Asian population
}

Chee Ping $\underline{C h e w^{1}}$, MBBS, MRCs, Kong Hwee $\underline{L} e^{1}$, MBBS, MRCs, Joyce Suang Bee $\underline{K o h}^{1}$, MBBS, FRCS, Tet Sen $\underline{\text { Howe }}^{1}$, FRCS, FAMS

INTRODUCTION The fabella, a sesamoid bone sometimes found in the lateral head of the gastrocnemius muscle, often articulates directly with the lateral femoral condyle. This study aimed to determine the incidence of fabellae in an Asian population and to characterise the radiological features of the fabella.

METHODS Electronic radiographs and magnetic resonance imaging films of 80 consecutive patients who underwent knee arthroscopy between May 2005 and October 2009 were reviewed to determine the presence and characteristics of the fabella.

RESULTS The incidence of fabellae was $31.25 \%$ in our study cohort. The median length, thickness, width and distance of the fabella from the lateral femoral condyle were $7.06 \mathrm{~mm}, 4.89 \mathrm{~mm}, 6.12 \mathrm{~mm}$ and $33.19 \mathrm{~mm}$, respectively. The fabella was consistently bony and located in the lateral head of the gastrocnemius, with $52 \%$ of the fabellae having an articulating facet. Fabellae in men were found to be larger than in women, although the difference was not statistically significant. The presence of an articulating groove was associated with increased size of the fabella, but not with the distance between the fabella and its insertion onto the lateral head of the gastrocnemius.

CONCLUSION The incidence of fabellae in our population was lower than that in regional studies. They were consistently bony and not all had articulating grooves on the lateral femoral condyle. We found that the larger the fabella, the higher the chances of it having an articulating groove. By defining the radiological characteristics of the fabella, we provide objective parameters to help differentiate the fabella from other loose bodies or calcifications in the knee.

Keywords: fabella, knee joint, sesamoid bones

\section{INTRODUCTION}

The fabella (or 'little bean') is a sesamoid bone occasionally found in the tendinous portion of the lateral head of the gastrocnemius muscle, frequently articulating directly with the lateral femoral condyle. ${ }^{(1)}$ In domestic animals such as cats and dogs, a sesamoid bone in the gastrocnemius muscle is consistently present. ${ }^{(2,3)}$ In humans, however, the fabella is not always present, with the reported incidence ranging between $8.7 \%$ and $31.3 \%$ in western populations, ${ }^{(4,5)}$ and between $66.0 \%$ and $85.8 \%$ in Japanese populations. ${ }^{(6,7)}$

Various cadaveric studies on the fabella have described its anatomy, relationship with the surrounding tissues, and clinical implications. ${ }^{(5)}$ Imaging studies, mainly case reports, have described the fabella in relation to clinical scenarios such as osteoarthritis ${ }^{(6)}$ and pain following total knee arthroplasty. ${ }^{(8)}$ However, those imaging studies did not adequately describe the radiological characteristics of the fabella. The present study aimed to determine the incidence of fabellae in an Asian population, as well as characterise the radiological features of the fabella.

\section{METHODS}

We retrospectively reviewed the electronic and hard copy medical records of 80 consecutive patients who underwent therapeutic arthroscopy of the knee under two senior orthopaedic consultants at the Department of Orthopaedic Surgery, Singapore General Hospital, Singapore, between May 2005 and October 2009. The patients' biodata and operative details were retrieved. The electronic radiographs and magnetic resonance (MR) imaging films of the knee were reviewed using the Image Management System (IMS) (Carestream Health, New York, USA). Great care was exercised in the documentation of the presence of a fabella (both bony and cartilaginous). Generally, radiographs of the knee would be able to detect bony fabellae, whereas MR imaging of the knee would detect both bony and cartilaginous fabellae. Areas of ectopic calcification or ossification were identified and excluded. Care was taken not to confuse the fabella with intra-articular loose bodies, especially on anteroposterior projection of the knee on radiographs. This differentiation is generally best achieved on lateral radiographs that show the intratendinous position of the fabella, as well as its fixed position during dynamic examination..$^{(9)}$

In patients where a fabella was present, its parameters were documented based on MR imaging measurements of the cuts of the knee (sagittal/coronal/axial view), using the tools available in the IMS software package. Documented parameters of the fabella included maximum length (Fig. 1), maximum thickness (Fig. 2), maximum width (Fig. 3), distance between the fabella and the insertion of the lateral head of the

${ }^{1}$ Department of Orthopaedic Surgery, Singapore General Hospital, Singapore

Correspondence: Dr Chew Chee Ping, Registrar, Department of Orthopaedic Surgery, Singapore General Hospital, Outram Road, Singapore 169609. bobchew_79@hotmail.com 


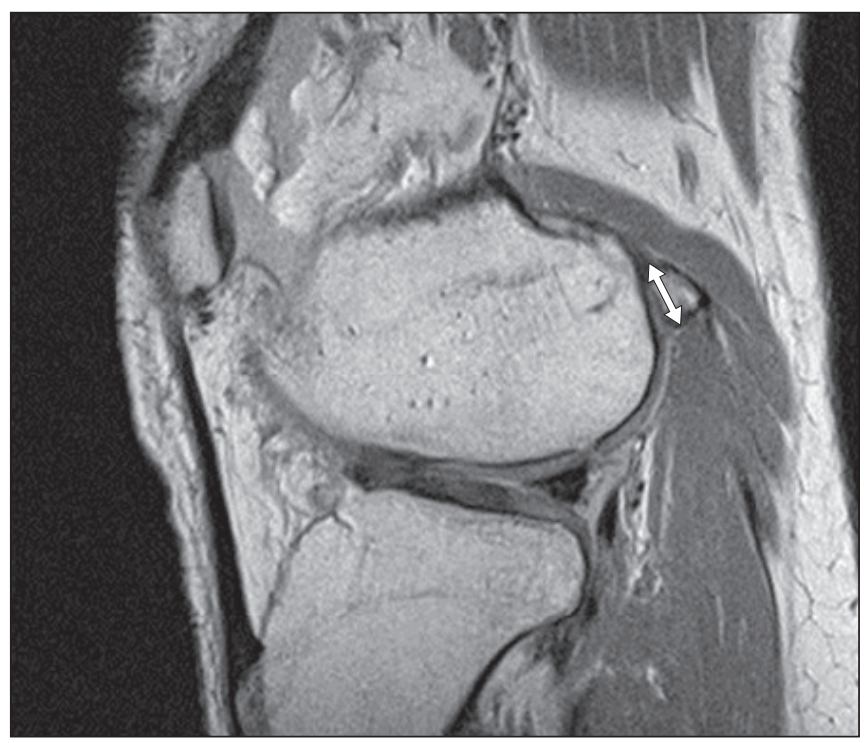

Fig. 1 Sagittal MR image of the knee shows the measurement of the maximum length of the fabella (double-headed arrow).

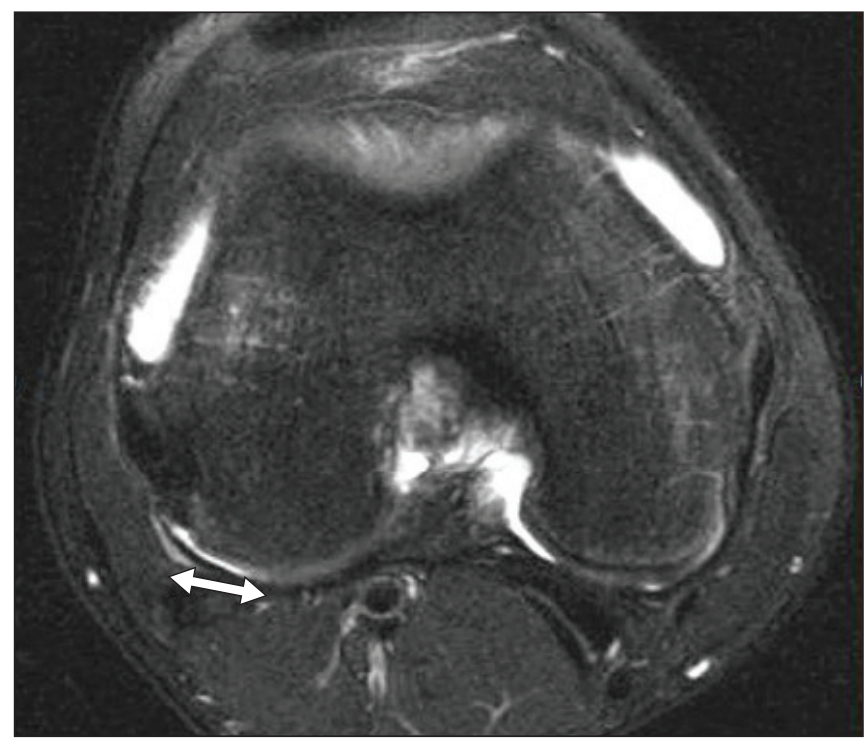

Fig. 3 Axial MR image of the knee shows the measurement of the maximum width of the fabella (double-headed arrow).

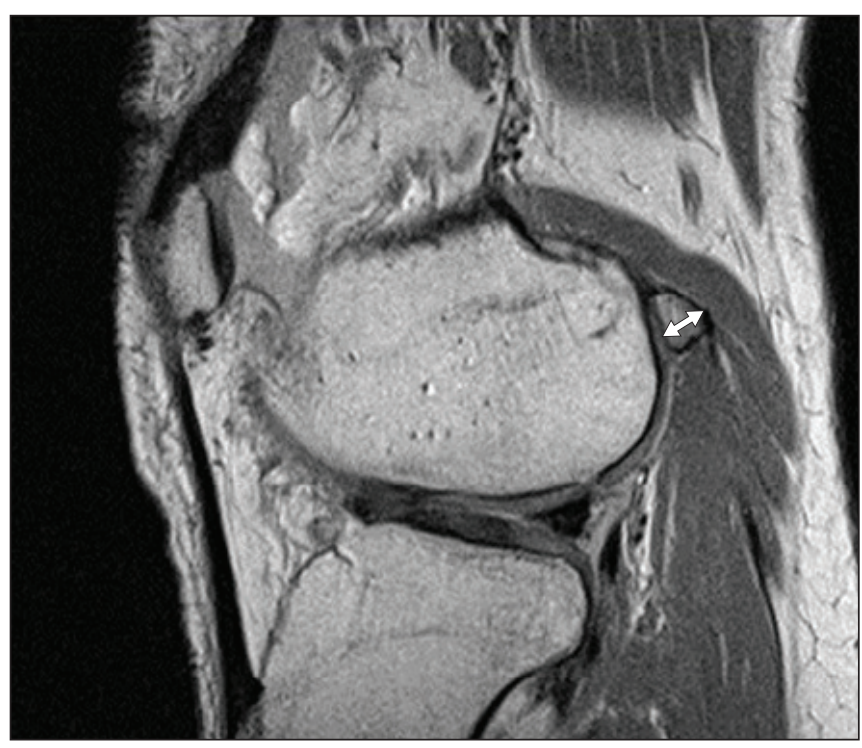

Fig. 2 Sagittal MR image of the knee shows the measurement of the maximum thickness of the fabella (double-headed arrow).

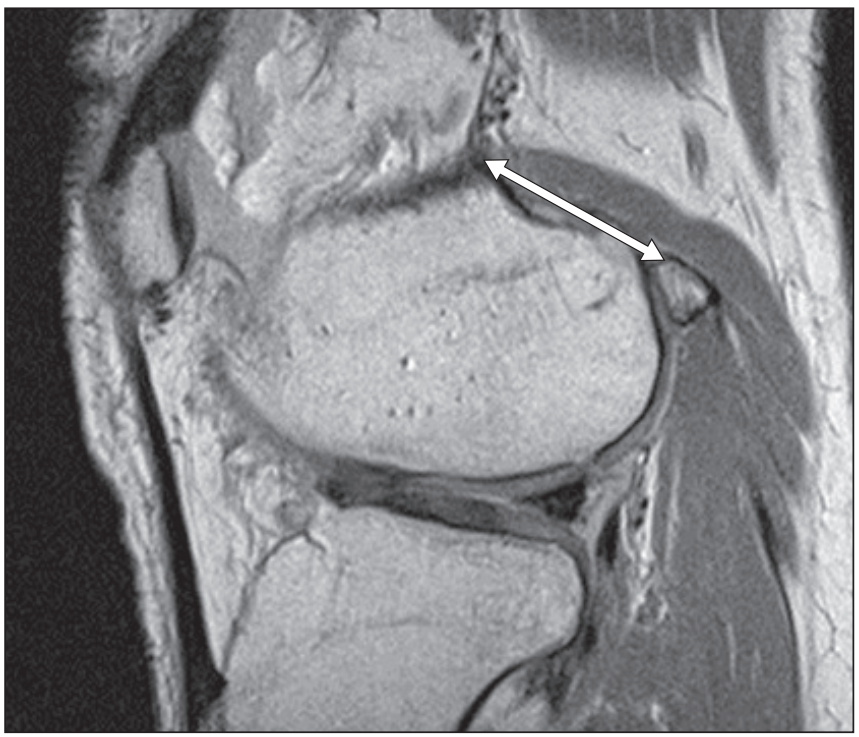

Fig. 4 MR image of the knee shows the measurement of the distance between the fabella and the insertion of the lateral head of the gastrocnemius onto the femur (double-headed arrow).
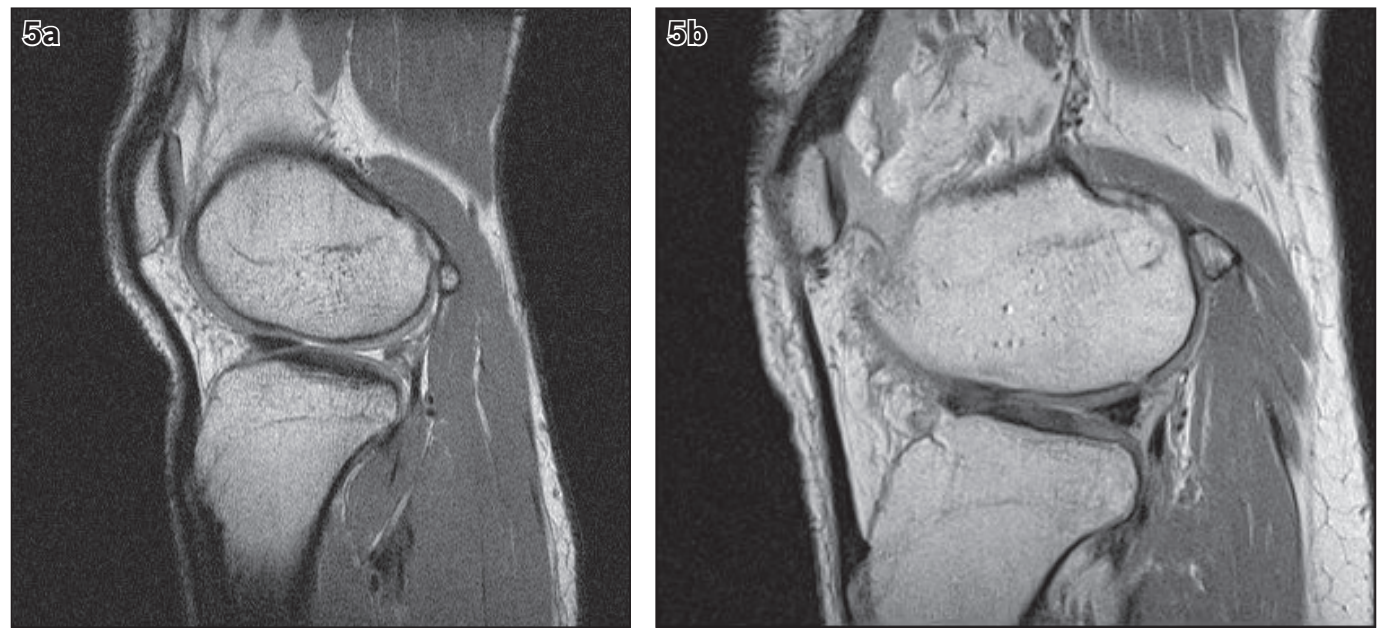

Fig. 5 MR images of the knee show (a) concave and (b) flat contours of the articulating facet of the lateral femoral condyle of the femur. 
Table I. Parameters of the fabella with respect to gender.

\begin{tabular}{lcccc}
\hline Parameter & \multicolumn{1}{c}{ Mean \pm SD (range) } & W-value \\
\cline { 2 - 4 } & Overall & Men & $5.86 \pm 0.99$ & \\
\hline Length $(\mathrm{mm})$ & $7.06 \pm 1.90$ & $7.50 \pm 1.91$ & $(5.16-6.56)$ & 0.280 \\
& $(4.96-11.29)$ & $4.69-11.29)$ & $(3.65 \pm 1.94$ & 0.667 \\
Thickness $(\mathrm{mm})$ & $4.89 \pm 1.94$ & $(2.93-8.91)$ & $5.49 \pm 0.21$ & $0.02)$ \\
& $(2.93-8.91)$ & $6.95 \pm 3.35$ & $(5.34-5.63)$ & 0.373 \\
Width $(\mathrm{mm})$ & $6.12 \pm 3.26$ & $(3.00-15.96)$ & $30.93 \pm 0.02$ & 0.060 \\
& $(3.00-15.96)$ & $34.04 \pm 4.32$ & $(31-31)$ & \\
Distance from the fabella to & $33.19 \pm 4.33$ & $(30-44)$ & & \\
the insertion of the LG $(\mathrm{mm})$ & $(30-44)$ & &
\end{tabular}

LG: lateral head of the gastrocnemius; SD: standard deviation

Table II. Parameters of the fabella with respect to the presence of an articulating groove.

\begin{tabular}{lcr}
\hline Parameter & \multicolumn{1}{c}{ Mean \pm SD (range) } & \multicolumn{1}{c}{$\begin{array}{c}\text { Absence of } \\
\text { articulating groove }\end{array}$} \\
\cline { 2 - 3 } & $\begin{array}{c}\text { Presence of } \\
\text { articulating groove }\end{array}$ & $6.14 \pm 1.51(5.16-6.56)$ \\
\hline Length $(\mathrm{mm})$ & $8.12 \pm 1.77(6.00-11.29)$ & $3.80 \pm 0.98(2.93-6.02)$ \\
Width $(\mathrm{mm})$ & $6.70 \pm 1.67(4.14-8.91)$ & $4.65 \pm 1.94(3.28-6.02)$ \\
Thickness $(\mathrm{mm})$ & $6.70 \pm 1.97(2.93-8.91)$ & $33.61 \pm 4.14(30.00-43.00)$ \\
Distance from the fabella to & $32.00 \pm 4.59(32.00-44.00)$ & 0.470 \\
the insertion of the LG $(\mathrm{mm})$ & & \\
\hline$*$
\end{tabular}

${ }^{*} p<0.05$ was considered statistically significant. LG: lateral head of the gastrocnemius; SD: standard deviation

gastrocnemius onto the femur (Fig. 4), and the presence of any articulating facet. The presence of an articulating facet was defined as either a flat or concave contour of the lateral femoral condyle (Fig. 5). Statistical analysis was performed using Pearson's chi-square test and Mann-Whitney $U$ test. A p-value of $<0.05$ was considered statistically significant.

\section{RESULTS}

The average age of the 80 patients who underwent arthroscopy of the knee was 28.4 (range 14-55) years. A majority of the patients were men (72 [90\%] men; 8 [10\%] women). Among the 80 patients, 30 had meniscal injuries (e.g. lateral or medial meniscus tears or degeneration) with intact knee cruciate ligaments and 50 had ligamentous injuries of the knee with or without associated meniscal injuries. Of these 50 patients with ligamentous injuries, 42 had complete tear of the anterior cruciate ligament $(\mathrm{ACL})$, one had tear of an $A C L$ graft, three had single bundle tear of the $A C L$, two had posterior cruciate ligament $(\mathrm{PCL})$ tears, and two had both $\mathrm{ACL}$ and PCL tears.

The presence of a fabella was noted in 25 patients (23 [92\%] men; 2 [8\%] women), indicating an overall incidence of $31.25 \%$ in our study group. There was no statistically significant difference in the incidence of fabella between men and women $(p=0.688)$. In our cohort, the fabellae were all bony (i.e. fabella that was detectable on both radiography and MR imaging, of the knee). There was no cartilaginous fabella detected (i.e. fabella that could be identified on MR imaging, but not on radiography). All the fabellae were located within the lateral head of the gastrocnemius, and no fabellae were noted in the medial head of the gastrocnemius.
Of the 25 fabellae, a majority ( $\mathrm{n}=16,64 \%$ ) was noted in knees with disrupted knee ligaments; only 9 (36\%) fabellae were observed in knees with intact knee ligaments. However, there was no statistically significant difference in the incidence of fabella between patients with intact knee ligaments and those with disrupted knee ligaments $(p=0.852)$. Among the 25 knees with fabellae, articulating facets were noted in 13 knees, indicating an incidence of $52 \%$ for articulating facets in our study. Of these articulating facets, 3 (23.1\%) facets were concave and $10(76.9 \%)$ were flat.

Overall, the median length, thickness and width of the fabella were $7.06 \mathrm{~mm}, 4.89 \mathrm{~mm}$ and $6.12 \mathrm{~mm}$, respectively (Table I). The median distance from the fabella to the insertion of the lateral head of the gastrocnemius onto the lateral femoral condyle was $33.19 \mathrm{~mm}$. Analysis of the parameters of the fabella according to gender showed that, in men, the median length of the fabella was $7.50 \mathrm{~mm}$, median thickness was $4.89 \mathrm{~mm}$, median width was $6.95 \mathrm{~mm}$, and median distance from the fabella to the insertion of the lateral head of the gastrocnemius was $34.04 \mathrm{~mm}$. In women, the corresponding measurements were $5.86 \mathrm{~mm}, 4.65 \mathrm{~mm}, 5.49 \mathrm{~mm}$ and $30.93 \mathrm{~mm}$.

Generally, the fabella in men were found to be consistently larger than that in women; the fabella in men also showed a correspondingly wider range of variations. However, statistical analysis using the Mann-Whitney $U$ test revealed no significant difference between the genders. The Mann-Whitney $U$ test showed a statistically significant relationship between the presence of an articulating groove and the length, thickness and width of the fabella. However, there was no such association between the presence of an articulating groove 
and the distance between the fabella and its insertion onto the lateral head of the gastrocnemius (Table II).

\section{DISCUSSION}

The fabella has been described as a sesamoid bone, a term that refers to small bones embedded in certain tendons and usually related to joint surfaces. Sarin et al have postulated functional and phylogenetic theories regarding the development of these bones. ${ }^{(5)}$ According to phylogenetic theory, genetic intrinsic factors that develop during the evolutionary process lead to the development of sesamoid bones. These appear in the womb and are initially cartilaginous. After birth, the bones calcify, depending on the kind of activity performed by the individual. The functional theory, on the other hand, suggests that the fabella is present because it helps the joint gain a biomechanical advantage by functioning as a pulley, reducing the friction of the tendons and potentiating the muscular handspike.

While the incidence of fabellae in our study (31.25\%) was within the ranges specified in the literature for western populations, ${ }^{(4,5)}$ it was much lower than the incidences reported by Japanese studies. ${ }^{(6,9)}$ This difference could be attributed to the inherent cultural differences between the populations studied. For instance, tatami mats are a deeply rooted tradition in Japanese culture, and the Japanese people have a predilection for kneeling on these mats during social functions. According to phylogenetic theory, the persistent presence and pressure of the fabella against the posterior aspect of the lateral femoral condyle under such circumstances might spur the development and ossification of the fabella.

Similar to previous studies, ${ }^{(4,6,9)}$ we did not find any statistically significant difference in the incidence of fabellae between men and women. However, in our cohort, the fabellae were consistently larger in men than in women. As men have larger body masses and muscles than women, the presence of a larger fabella might theoretically help the knee joint gain a biomechanical advantage. While our analysis did not show any significant difference between the two genders with respect to the size of the fabella, it is possible that our findings were biased due to the small sample size of women with fabellae.

Several anatomical studies have described the fabella to be either cartilaginous or bony. ${ }^{(4,6)}$ Kawashima et al, for instance, noted that fabellae in the lateral head of the gastrocnemius were mostly cartilaginous $57.3 \%$ cartilaginous vs. $34.7 \%$ bony). ${ }^{(6)}$ However, in our study, the fabellae were consistently bony, with no cartilaginous fabellae found. It is well known that the human patella - also a sesamoid bone - normally forms at around 9-10 weeks of life, starts to ossify from three years of age and is completely ossified by the age of 20 years. ${ }^{(5)}$ Our findings suggest that the fabella is similar to the patella in this regard, with ossification occurring as early as 14 years of age.

Kawashima et al also reported that bony fabellae consistently make an impression on the lateral femoral condyle. ${ }^{(6)}$ However, in our study, an articular groove on the lateral femoral condyle was not always present, with only $52 \%$ of the bony fabellae having a corresponding articular groove on the lateral femoral condyle. We found that the larger the fabella, the higher the likelihood of it having an articular facet.

It has been suggested that the size and position of the fabella play a role in the development of symptoms, with some studies reporting fabellae measuring 4-22 $\mathrm{mm}$ in symptomatic patients. ${ }^{(10,11)}$ It has also been suggested that a fabella larger than $10 \mathrm{~mm}$ in size requires excision in patients undergoing total knee replacement. ${ }^{(12)}$ However, Weiner and Macnab recommend a six-month period during which conservative management of symptomatic fabellae should be attempted prior to the consideration of surgery for patients with fabella syndrome. ${ }^{(13)}$ Conservative management generally includes steroid injections, immobilisation, analgesics and restricted activities. The potential role of prophylactic arthroscopic fabellectomy in patients with a fabella larger than $10 \mathrm{~mm}$ and undergoing therapeutic arthroscopy is an aspect that could be further explored in subsequent studies.

In conclusion, the incidence of fabellae in our Asian population was within the range specified for western populations but lower than the ranges reported in Japanese studies. Our findings lend support to the phylogenetic theory for the formation of the fabella. The fabellae in our study were consistently bony, but not all had articulating grooves on the lateral femoral condyle, in contrast to some regional cadaveric studies. Only large fabellae were associated with articular grooves in our population. Our study delineates the radiological characteristics of the fabella and provides objective parameters that could help to differentiate the fabella from other loose bodies or calcifications in the knee.

\section{REFERENCES}

1. Duncan W, Dahm DL. Clinical anatomy of the fabella. Clin Anat 2003; 16:448-9.

2. Crouch JE. Text-atlas of cat anatomy. Philadelphia: Lea \& Febiger, 1969.

3. Miller ME, Evans HE, Christensen GC. Miller's anatomy of the dog. 2nd ed. Philadelphia: Saunders, 1979: 205-15.

4. Kaplan EB. The fabellofibular and short lateral ligaments of the knee joint. J Bone Joint Surg Am 1961; 43:169-79.

5. Sarin VK, Erickson GM, Giori NJ, Bergman AG, Carter DR. Coincident development of sesamoid bones and clues to their evolution. Anat Rec 1999; 257:174-80.

6. Kawashima T, Takeishi $\mathrm{H}$, Yoshitomi S, Ito M, Sasaki H. Anatomical study of the fabella, fabellar complex and its clinical implications. Surg Radiol Anat 2007; 29:611-6.

7. Minowa T, Murakami G, Kura H, et al. Does the fabella contribute to the reinforcement of the posterolateral corner of the knee by inducing the development of associated ligaments? J Orthop Sci 2004; 9:59-65.

8. Pritchett JW. The incidence of fabellae in osteoarthrosis of the knee. J Bone Joint Surg Am 1984; 66:1379-80.

9. Davies AM, Cassar-Pullicino VN, eds. Imaging of the Knee: Techniques and Applications. Berlin: Springer, 2003.

10. Mangieri JV. Peroneal-nerve injury from an enlarged fabella. A case report. J Bone Joint Surg Am 1973; 55:395-7.

11. Takebe K, Hirohata K. Peroneal nerve palsy due to the fabella. Arch Orthop Trauma Surg 1981; 99:91-5.

12. Wang JW. Fabellar impingement after total knee replacement - a case report. Changgeng Yi Xue Za zhi 1995; 18:185-9.

13. Weiner DS, Macnab I. The "fabella syndrome": an update. J Pediatr Orthop 1982; 2:405-8 